\title{
Übersichtlichkeit als Mediator zwischen Ästhetik und Usability?
}

\author{
Martin Schrepp, Kerstin Eva Müller
}

SAP AG

\section{Zusammenfassung}

Ein gut untersuchtes Phänomen ist der Zusammenhang zwischen wahrgenommener Ästhetik und Usability einer Nutzungsschnittstelle. Mehrere Untersuchungen zeigten einen positiven Einfluss der Ästhetik auf die Usability. Aber auch für einen umgekehrten Zusammenhang gibt es empirische Belege. Als Erklärung wurden bisher ein Einfluss der Stimmung des Nutzers oder das aus der Sozialpsychologie bekannten Attraktivitätsstereotyp vorgeschlagen. Wir untersuchen eine weitere Erklärungsmöglichkeit, die auf der Beobachtung basiert, dass manche Items in UX Fragebögen klassischen Usability Dimensionen zugeordnet sind, während dieselben oder sehr ähnliche Items in anderen Fragebögen als Indikator für Ästhetik verwendet werden. Eine inhaltliche Analyse dieser Items deutet auf ein Konzept der wahrgenommenen Übersichtlichkeit hin, dass die subjektive Wahrnehmung von Usability und Ästhetik gleichermaßen beeinflusst. Daten aus einer größeren Online-Studie scheinen diese Annahme zu stützen.

\section{Einleitung}

Erfolgreiche Produkte müssen sowohl einfach zu bedienen sein, als auch ein attraktives Design aufweisen. Die Forschung im Bereich Mensch-Maschine-Interaktion hat sich ursprünglich sehr stark auf die Qualität der Bedienung fokussiert. Hier standen klassische Usability Dimensionen (z.B. Effizienz, Erlernbarkeit oder Fehlertoleranz) klar im Mittelpunkt des Interesses. In den letzten Jahren hat sich hier ein Wechsel zu einer holistischen Sicht der User Experience ergeben, die auch hedonische Qualitäten eines Produkts (Hassenzahl 2001, 2004) berücksichtigt, wie z.B. Ästhetik, Spass bei der Nutzung oder Stimulation.

Eine natürliche Fragestellung in diesem Rahmen ist, in welcher Beziehung hedonische und pragmatische Qualitäten einer Nutzungsschnittstelle stehen. Mehrere Untersuchungen, z.B. Kurosu \& Kashimura (1995), Tractinsky (1997) und Tractinsky et al. (2000), zeigten einen positiven Einfluss der wahrgenommenen Ästhetik auf die wahrgenommene Usability, was oft kurz und prägnant als What is beautiful is usable formuliert wird. 
Wodurch kann dieser Einfluss der wahrgenommenen Ästhetik auf die wahrgenommene Usability erklärt werden? Hierfür wurden bisher im Wesentlichen zwei Erklärungen vorgeschlagen und auch empirisch untersucht.

Ein erster Erklärungsansatz basiert auf dem vermittelnden Einfluss der Stimmung des Nutzers auf wahrgenommene Usability und Ästhetik. Ergebnisse von Isen (2000) zeigen, dass ein positiver emotionaler Zustand einer Person deren Kreativität und Flexibilität beim Problemlösen erhöht. Ein negativer emotionaler Zustand begünstigt dagegen eher einen systematisch-analytischen Umgang mit Problemen (Schwarz 2002). Bei der Interaktion mit einer Benutzungsschnittstelle sollte ein Nutzer in guter Stimmung auftretende Schwierigkeiten eher durch geeignete Heuristiken umgehen und daher als weniger schwerwiegend empfinden. Ein Nutzer in schlechter Stimmung wird sich dagegen eher auf problematische Details fokussieren. Daher sollte ein Nutzer in schlechter Stimmung die Usability einer Benutzungsschnittstelle eigentlich schlechter einschätzen als ein Nutzer in guter Stimmung.

Norman (2003) zieht diesen Effekt als Erklärung für den Zusammenhang zwischen wahrgenommener Usability und wahrgenommener Ästhetik heran. Er argumentiert, dass eine ästhetisch ansprechende Gestaltung den emotionalen Zustand des Nutzers verbessert. Diese verbesserte Stimmung sollte dann über den oben beschriebenen Mechanismus zu einer besseren Bewertung der Usability führen. Die Stimmung des Nutzers fungiert in diesem Erklärungsansatz als Mediator zwischen Ästhetik und Usability.

Eine andere häufig herangezogene Erklärungsmöglichkeit beruht auf dem aus der Sozialpsychologie gut bekannten Attraktivitäts-Stereotyp (Was schön ist, ist auch gut), dem sogenannten Halo-Effekt. Untersuchungen (siehe z.B. Dion et al. 1972 oder Dick et al. 1990) in diesem Bereich haben gezeigt, dass menschliche Schönheit mit Eigenschaften wie sozialer Kompetenz oder hoher Intelligenz in Zusammenhang gebracht wird. Aus der Marktforschung ist das Phänomen der evaluativen Konsistenz bekannt. Es geht hierbei um die Verhaltensweise, dass fehlende Produktinformationen aus der allgemeinen Bewertung einer Marke abgeleitet werden. Zum Beispiel wird oft aus einem hohen Preis auf eine hohe Qualität eines Produkts geschlossen (Ford \& Smith 1987).

Überträgt man dies auf die Bewertung von Nutzungsschnittstellen, so sollten fehlende Informationen zur Usability aus leicht sichtbaren und daher bekannten Informationen zur wahrgenommenen Ästhetik hergeleitet werden.

In einer Studie (Ilmberger et al. 2009) wurde untersucht, welcher dieser Erklärungsversuche geeigneter ist. Die Ergebnisse deuteten eher in Richtung der Annahme von Norman (2003), waren aber nicht eindeutig.

Eine Schwäche beider Erklärungsansätze ist, dass sie keine gute Begründung für den in mehreren Studien ebenfalls nachgewiesenen Einfluss von wahrgenommener Usability auf wahrgenommene Ästhetik liefern, kurz formuliert als What is usable is beautiful (siehe z.B. Ilmberger et al. 2009 oder Tuch et al. 2012).

Wir wollen im Folgenden eine weitere Erklärungsmöglichkeit für den Zusammenhang zwischen wahrgenommener Usability und Ästhetik untersuchen. Diese beruht auf der 
Annahme, dass eine gemeinsame Komponente existiert, die sowohl die Wahrnehmung der Ästhetik, als auch die Wahrnehmung der Usability beeinflusst. Dies würde den Zusammenhang in beiden Richtungen erklären. Im nächsten Abschnitt werden wir diese Erklärung genauer begründen.

\section{2 Übersichtlichkeit als Mediator}

Lavie und Tractinsky (2004) unterscheiden zwei Komponenten der wahrgenommenen Ästhetik von Web-Seiten, die sie als klassische und expressive Ästhetik bezeichnen. Klassische Ästhetik fokussiert auf Aspekte eines geordneten und klaren Designs. Expressive Ästhetik dagegen auf Kreativität und Originalität. Klassische Ästhetik wird in der oben zitierten Arbeit mit Begriffen wie klar (clear), organisiert (organized), übersichtlich (clean) oder symmetrisch (symmetrical) operationalisiert.

Auch im VISAWI Fragebogen (Moshagen \& Thielsch 2010) zur Messung der visuellen Ästhetik von Web-Seiten taucht dieses Konstrukt in einer der vier Dimensionen (Einfachheit) auf. Entsprechende Items sind hier Das Layout wirkt zu gedrängt, Das Layout ist gut zu erfassen und Das Layout scheint angenehm gegliedert.

Deng \& Poole (2012) konnten bei einer Untersuchung von E-Commerce Webseiten nachweisen, dass die zwei Faktoren Komplexität und Ordnung (im Sinne klassischer Ästhetik) einen wesentlichen Einfluss auf die wahrgenommene Ästhetik haben und die Präferenz für Webseiten beeinflussen. Dieses Ergebnis passt auch zu einer Theorie von Birkhoff (1933), die das Verhältnis von Ordnung und Komplexität als Maß für die ästhetische Qualität eines Objekts vorschlägt.

Nach Ngo et al. (2003) sind für eine Messung der Ästhetik Faktoren wie Balance, Symmetrie und Einfachheit zentral, d.h. Faktoren, die man dem Bereich der klassischen Ästhetik im Sinne von Lavie \& Tractinsky (2004) zuordnen kann.

Ein weiterer bekannter Effekt bei der Wahrnehmung von Ästhetik ist der Fluency-Effekt (Reber et al. 2004). Die Autoren zeigen, dass Objekte umso ästhetischer wahrgenommen werden, je leichter sie zu verarbeiten sind. Dies erklärt warum Objekteigenschaften wie Symmetrie und Übersichtlichkeit die wahrgenommene Ästhetik erhöhen.

Dieser Aspekt der Ästhetik zielt also auf ein klar gegliedertes, einfaches und damit gut zu erfassendes Layout. Wir bezeichnen diesen Aspekt im Folgenden als Übersichtlichkeit.

Items, die diesen Aspekt der Produktwahrnehmung beschreiben, finden sich in anderen Fragebögen allerdings auch als Indikatoren für klassische Usability Dimensionen. Der UEQ (Laugwitz et al. 2006, 2008) enthält ein Item aufgeräumt/überladen, dass dort der Dimension Effizienz zugeordnet ist und ein Item übersichtlich/verwirrend mit einer Zuordnung zur Dimension Durchschaubarkeit (Erlernbarkeit). Im AttraktDiff2 (Hassenzahl et. al 2003) findet sich in der Skala Pragmatische Qualität ein Item verwirrend / übersichtlich (ein weiteres ähnliches Item dieser Skala ist einfach / kompliziert). 
Also laden Items der Bedeutungsdimension übersichtlich/unübersichtlich offenbar auch auf klassischen Dimensionen der Usability. Betrachtet man dies näher, so ist eigentlich relativ klar, dass ein übersichtliches klar strukturiertes Layout die Effizienz des Nutzers bei der Orientierung auf der Benutzungsoberfläche erhöht. Zusätzlich impliziert ein solches Layout auch Einfachheit und erweckt zumindest den Eindruck, die entsprechende Nutzungsoberfläche schnell verstehen und erlernen zu können.

Es ist also plausibel, hier eine Ursache für den Zusammenhang zwischen wahrgenommener Usability und Ästhetik zu sehen. Ist eine Benutzerschnittstelle im Sinne der klassischen Ästhetik gut gestaltet, d.h. macht sie einen übersichtlichen, gut strukturierten Eindruck, sollte dies die wahrgenommene Ästhetik, aber auch die Einschätzung der Usability, positiv beeinflussen. Dies würde auch gut erklären, warum es für beide Richtungen (What is beautiful is usable und What is usable is beautiful) empirische Belege gibt. Wir nehmen also an, dass die Übersichtlichkeit einer Benutzerschnittstelle als Mediator den Zusammenhang zwischen Usability und Ästhetik erklären kann.

\section{Online-Studie}

Die im Folgenden beschriebene Online-Studie bestand aus drei unabhängig voneinander durchgeführten Teilstudien. Diese waren im Ablauf völlig identisch und unterschieden sich nur in den verwendeten Stimuli. Diese waren Startseiten des Web-Auftritts von Städten (Studie 1), Web-Shops (Studie 2) und Design-Agenturen (Studie 3). Pro Kategorie wurden 10 Web-Seiten ausgewählt.

\subsection{Teilnehmer}

Die Teilnehmer wurden über Gruppen in sozialen Netzwerken und über Online-Foren angeworben. Für die Teilnahme gab es keine Entlohnung. Insgesamt konnten die Daten von 423 Teilnehmern ausgewertet werden. Die Abbruchquote (Prozentsatz der Teilnehmer, die das Online-Experiment zwar gestartet, aber nicht beendet hatten) betrug über alle drei Teilstudien hinweg 40,3\%. Für die einzelnen Teilstudien ergaben sich folgende Teilnehmerzahlen und demographische Daten:

- Städte: 169 Teilnehmer, 44\% männlich, 43\% weiblich, 13\% ohne Angabe, mittleres Alter 31,75 Jahre

- Web-Shops: 101 Teilnehmer, 42\% männlich, 41\% weiblich, 17\% ohne Angabe, mittleres Alter 32,4 Jahre

- Agenturen: 153 Teilnehmer, 42\% männlich, 31\% weiblich, 27\% ohne Angabe, mittleres Alter 28,6 Jahre 


\subsection{Materialien}

Durch die 30 verwendeten Web-Seiten und die Auswahl aus drei unterschiedlichen Kategorien sollte ein möglichst breites Spektrum unterschiedlicher Seiten abgedeckt werden. Von den ausgewählten Seiten wurden Screen-Shots in der Auflösung 1024 x 768 gezogen, die dann in der Studie präsentiert wurden. Abbildung 1 zeigt aus jeder der drei verwendeten Kategorien jeweils zwei Screen-Shots.
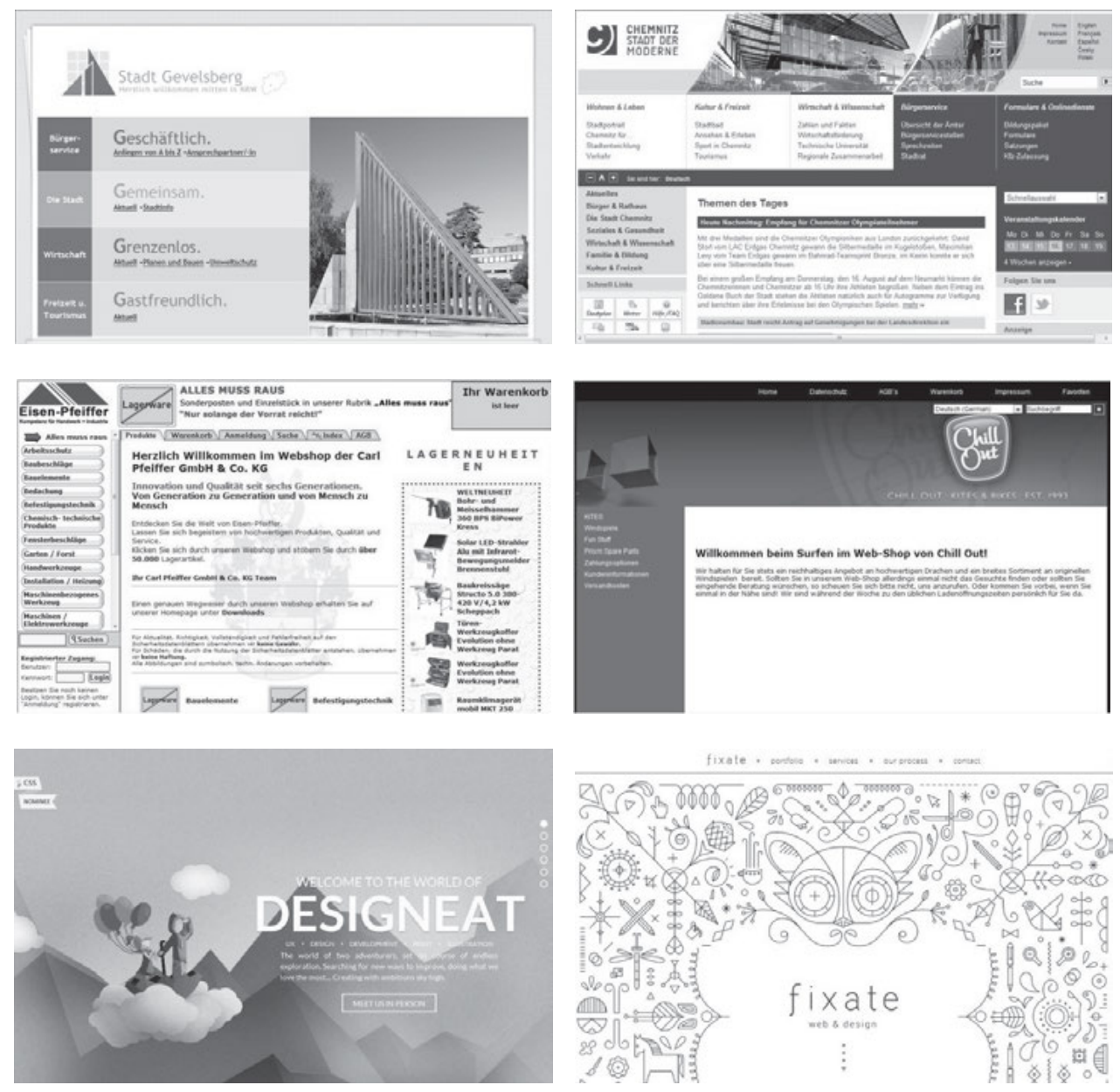

Abbildung 1: Beispiele verwendeter Screen-Shots aus der Studie.

\subsection{Ablauf}

Nachdem der Teilnehmer die Online-Studie aufgerufen hatte, wurde zunächst eine Seite mit allgemeinen Instruktionen zur Studie angezeigt. Danach wurde zufällig eine der 10 Einstiegsseiten als Screenshot dargeboten. Der Teilnehmer konnte diese so lange betrachten, 
wie er bzw. sie mochte. Unter dem Screenshot befand sich ein Online-Formular mit den Fragen:

- Die Seite ist schön.

- Ich denke, ich würde mit der Seite gut zurechtkommen.

- Die Seite ist übersichtlich.

- Zusätzlich wurden noch die vier Fragen aus der Kurzform des VISAWI (Thielsch \& Moshagen 2011) vorgegeben.

Anhand dieser Fragen konnte der Teilnehmer die Seite bewerten (7-stufige Likert-Skala pro Frage). Nach Ausfüllen dieses Formulars wurde die nächste zufällig gewählte Seite angeboten. Die Frage Ich denke, ich würde mit der Seite gut zurechtkommen wurde dabei als Indikator für die wahrgenommene Usability der Seite aufgefasst.

Jeder Teilnehmer beurteilte in dieser Weise drei zufällig ausgewählte Seiten (aus dem Vorrat der 10 ausgewählten Web-Auftritte pro Teilstudie). Ziel dieser Vorgehensweise war es, die Bearbeitungszeit für den Teilnehmer zu senken und dadurch die Abbruchquote gering zu halten. Allerdings waren dadurch nicht alle Seiten von der gleichen Zahl von Teilnehmern beurteilt (die Zahl der Urteile pro Seite variierte zwischen 22 und 59).

\section{Ergebnisse}

Die Studie sollte untersuchen, ob die wahrgenommene Übersichtlichkeit einer Web-Seite sowohl die wahrgenommene Usability als auch die wahrgenommene Ästhetik beeinflusst und auch als Erklärung des Zusammenhangs zwischen diesen beiden Komponenten dienen kann. Die Einschätzung der Schönheit korrelierte extrem hoch mit dem Ergebnis der Kurzversion des VISAWI $(r=0,80)$.

Die hochsignifikanten Korrelationen zwischen den untersuchten Variablen Usability, Ästhetik und Übersichtlichkeit betrugen:

- Usability, Ästhetik: $\mathrm{r}=0,44 \quad(\mathrm{t}(1072)=16,24, \mathrm{p}<0,001)$

- Übersichtlichkeit, Usability: $\mathrm{r}=0,71 \quad(\mathrm{t}(1072)=33,39, \mathrm{p}<0,001)$

- Übersichtlichkeit, Ästhetik: $\mathrm{r}=0,51 \quad(\mathrm{t}(1072)=19,18, \mathrm{p}<0,001)$

Betrachtet man die partielle Korrelation zwischen Usability und Ästhetik unter Kontrolle der Übersichtlichkeit, so ergibt sich nur ein Wert von 0,138 (signifikant, $\mathrm{t}(1072)=4,57$, p < 0,001). Berücksichtigt man also den Einfluss der Übersichtlichkeit auf die beiden anderen Variablen, so reduziert sich deren Zusammenhang deutlich. Dies ist ein erster Hinweis auf das Vorliegen eines vermittelnden Einflusses der Übersichtlichkeit auf Usability und Ästhetik. Für eine genauere Untersuchung des Zusammenhangs führen wir zwei MediatorAnalysen durch. 
Die Semi-Partiellen Korrelationen unter Kontrolle der Übersichtlichkeit betragen:

- Usability und Ästhetik: 0,09 ( $\mathrm{t}(1072)=3,18, \mathrm{p}<0,001)$

- $\quad$ Ästhetik und Usability: 0,12 ( $\mathrm{t}(1072)=3,93, \mathrm{p}<0,001)$

Betrachten wir zunächst den Einfluss von Ästhetik auf Usability. Die Ergebnisse der Mediator-Analyse sind in der oberen Hälfte von Abbildung 2 veranschaulicht. Die Werte ohne Klammern entsprechen den Regressionskoeffizienten der einfachen Regression von Ästhetik auf Usability, Übersichtlichkeit auf Usability und Ästhetik auf Usability. Die Werte in Klammern stellen die Regressionskoeffizienten der gemeinsamen Regression von Ästhetik und Übersichtlichkeit auf Usability dar (alle Zusammenhänge sind signifikant mit $\mathrm{p}<0,01$ ). Wie leicht zu sehen ist, verringert sich der Einfluss von Ästhetik auf Usability massiv, wenn man die Mediator-Variable Übersichtlichkeit berücksichtigt. Der Sobel-Test (Sobel 1982) zeigt ebenfalls einen signifikanten Mediator-Effekt an (Sobel z = 17,05, p < 0,01).

Betrachten wir nun den Einfluss von Usability auf Ästhetik. Die entsprechenden Ergebnisse der Mediator-Analyse sind im unteren Teil von Abbildung 2 dargestellt. Hier ergibt sich ebenfalls ein signifikanter Mediator-Effekt (Sobel z = 16,78, $\mathrm{p}<0,01$ ).

\section{Diskussion}

Für den Zusammenhang zwischen wahrgenommener Usability und Ästhetik einer Benutzerschnittstelle wurden bisher verschiedene Erklärungsmuster vorgeschlagen, z.B. der vermittelnde Einfluss der Stimmung des Nutzers oder das Attraktivitäts-Stereotyp. In diesem Beitrag wird versucht, diesen Zusammenhang über die wahrgenommene Übersichtlichkeit zu erklären. Dieser Erklärungsansatz ist deutlich einfacher als die oben genannten Ansätze und auch in der Lage den Zusammenhang in beiden Richtungen zu erklären (What is beautiful is usable und What is usable is beautiful). Aktuelle Theorien zur ästhetischen Wahrnehmung von Web-Seiten und die Analyse von existierenden Fragebögen zur Messung von User Experience lassen eine solche Erklärung auch theoretisch plausibel erscheinen.

In einer größeren Online Studie konnte ein deutlicher Mediator-Effekt der Übersichtlichkeit in beiden Richtungen der Beziehung zwischen Ästhetik und Usability nachgewiesen werden. Dieser erklärt den Zusammenhang allerdings nicht vollständig, d.h. es liegt eine partielle, aber keine vollständige Mediation vor. Eine spannende noch offene Frage ist, wie ein Konzept wie die wahrgenommene Übersichtlichkeit mit den bisher vorgeschlagenen Mediator-Variablen zusammenspielt. 

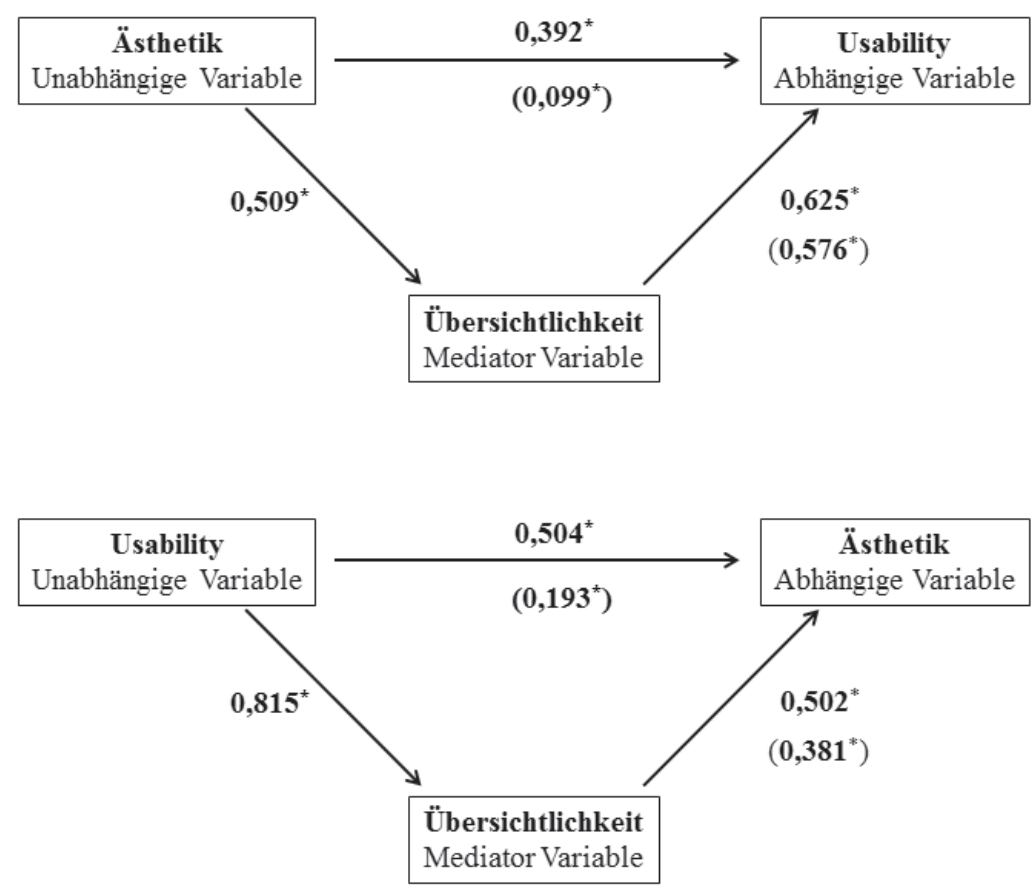

Abbildung 2: Zusammenhang zwischen Usability und Ästhetik mit Übersichtlichkeit als Mediator.

Unsere Studie liefert zumindest einen deutlichen ersten Hinweis, dass eine gemeinsame Komponente existiert, die die Wahrnehmung der Usability und Ästhetik einer Web-Seite gleichermaßen beeinflusst und was diese gemeinsame Komponente sein könnte. Es gibt natürlich eine Reihe von möglichen Störvariablen, die die Schlussfolgerungen unserer Studie möglicherweise beeinflusst haben und auf die wir jetzt noch eingehen wollen.

Eine mögliche Schwäche unserer Studie ist, dass die Teilnehmer Übersichtlichkeit, Ästhetik und Usability einer Web-Seite nur anhand von Screenshots beurteilten, d.h. nicht vorher mit der Web-Seite interagieren konnten. Besonders was die Einschätzung der Usability angeht, wurde in der vorliegenden Studie wegen der fehlenden Interaktion eher die Erwartung der Usability als die tatsächliche Usability gemessen. Dies hat möglicherweise einen Einfluss auf die Ergebnisse. In einer Studie von Ilmberger et al. (2009) zeigten sich z.B. deutliche Abweichung in der Einschätzung einiger User Experience Dimensionen vor und nach der Interaktion mit einem Web-Shop. Auch die vergleichsweise geringe Auflösung der Screenshots von 1024 x 768 könnte hier eine Auswirkung haben. Insofern wäre es wichtig, die Ergebnisse der Studie in Situationen zu replizieren, in denen die Teilnehmer wirklich für eine gewisse Zeit mit den beurteilten Web-Seiten gearbeitet haben.

Eine weitere Einschränkung, deren potentieller Einfluss auf die Ergebnisse in weiteren Studien noch genauer untersucht werden sollte, liegt in der Operationalisierung der 
wahrgenommenen Usability. Diese wurde nur durch eine Frage Ich denke ich würde mit der Seite gut zurechtkommen erhoben. Im Rahmen unserer Studie wurde auf weitere Fragen verzichtet, um die Zahl der Fragen pro Seite klein zu halten (da die Teilnehmer mehrere Web-Seiten beurteilen sollten, wäre ansonsten mit einer zu hohen Abbruchrate zu rechnen).

Allerdings ist es wünschenswert in einer Folgestudie (in der Teilnehmer dann evtl. nur eine Web-Seite beurteilen) einen etablierten Usability Fragebogen zu verwenden und dessen Ergebnisse dann mit den erhobenen Werten für Übersichtlichkeit und Ästhetik in Beziehung zu setzen. Für Ästhetik ist dieses Problem nicht so ausgeprägt, da zusätzlich zur Frage Die Seite ist schön noch die Kurzform des VISAWI vorgegeben wurde und die hohe Korrelation zwischen beiden Werten nahelegt, dass diese eine Frage das ästhetische Empfinden bereits gut abgebildet hat.

Visuelle Komplexität ist aus Sicht des Designers deutlich einfacher zu gestalten als Ästhetik oder Usability. Eine geringe wahrgenommene Komplexität lässt sich im Wesentlichen durch eine möglichst geringe Zahl von Elementen auf der Benutzerschnittstelle und gutes Alignment dieser Elemente erreichen (siehe z.B. Müller \& Schrepp 2013). Unsere Ergebnisse zeigen, wie wichtig diese einfachen Gestaltungskriterien für die wahrgenommene Usability und Ästhetik sind.

\section{Literaturverzeichnis}

Birkhoff G. D. (1933). Aesthetic Measure, Harvard University Press, Cambridge, MA.

Deng, L., \& Poole, M. S. (2012). Aesthetic design of e-commerce web pages - Webpage Complexity, Order and preference. Electronic Commerce Research and Applications 11(4), 420-440.

Dick, A., Dipankar C. \& Gabriel, B. (1990). Memory-Based Inference During Consumer Choice. Journal of Consumer Research, 17, S. 82-93.

Dion, K. K., Berscheid, E. \& Walster, E. (1972). What is beautiful is good. Journal of Personality and Social Psychology 24, S. 285-290.

Ford, G.T. \& Smith, R.A. (1987): Inferential Beliefs in Consumer Evaluations: An Assessment of Alternative Processing Strategies. Journal of Consumer Research 14, S. 363-371.

Hassenzahl, M. (2001). The effect of perceived hedonic quality on product appealingness. International Journal of Human-Computer Interaction 13(4), S. 481-499.

Hassenzahl, M. (2004). The Interplay of Beauty, Goodness and Usability in Interactive Products. Human-Computer Interaction 19, S. 319-349.

Hassenzahl, M.; Burmester, M.; Koller, F. (2003): AttrakDiff: Ein Fragebogen zur Messung wahrgenommener hedonischer und pragmatischer Qualität. In: J.Ziegler; G. Szwillus (Hrsg.): Mensch \& Computer 2003. Stuttgart: Teubner. S. 187-196.

Ilmberger, W.; Schrepp, M.; Held, T. (2009). Was verursacht den Zusammenhang zwischen Ästhetik und Usability. In: H. Wandke; S. Kain \& D. Struve (Eds.): Mensch \& Computer 2009. Oldenbourg Verlag, S. 383-392.

Isen, A. M. (2000). Positive affect and decision making. In Lewis, M., Haviland, J.M. (Hrsg.): Handbook of emotions ( $2^{\text {nd }}$ edtion), New York: Guilford Press, S. 417-435. 
Kurosu, M., \& Kashimura, K. (1995). Apparent usability vs. inherent usability: experimental analysis of the determinants of the apparent usability. Denver, Colorado: Conference Companion of human factors in computing systems, S. 292-293.

Laugwitz, B., Held, T. \& Schrepp, M. (2008). Construction and Evaluation of a User Experience Questionnaire. In Holzinger, A. (Hrsg.): HCI and Usability for Education and Work, LNCS 5298, Berlin, Heidelberg: Springer, S. 63-76.

Lavie, T. \& Tractinsky, N. (2004). Assessing dimensions of perceived visual aesthetics of web sites. International Journal of Human-Computer-Studies 60, S. 269-298.

Moshagen, M. \& Thielsch, M. T. (2010). Facets of visual aesthetics. International Journal of HumanComputer Studies 68, S. 689-709.

Müller, K. \& Schrepp, M. (2013). Visuelle Komplexität, Ästhetik und Usability von Benutzerschnittstellen. In: Boll, S.; Maaß, S. \& Malaka, R. (Eds.), Mensch \& Computer 2013, S 211 - 220, München: Oldenbourg Verlag.

Ngo, D. C., Teo, L. S., \& Byrne, J. G. (2000). Formalising guidelines for the design of screen layouts. Displays 21(1), 3-15.

Norman, D. (2003). Emotional Design: Why We Love (Or Hate) Everyday Things. Boulder Colorado: Basic Books.

Reber, R., Schwarz, N., \& Winkielman, P. (2004). Processing Fluency and Aesthetic Pleasure: Is Beauty in the Perceiver's Processing Experience? Personality and Social Psychology Review 8(4), 364-382.

Schwarz, N. (2002). Situated cognition and the wisdom of feelings. In Feldman-Barrett, L., Salovey, P. (Hrsg.): The wisdom of feeling: Psychological processes in emotional intelligence, New York: Guilford Press, S. 144-166.

Sobel, M. (1982). Asymptotic confidence intervals for indirect effects in structural equation modelling. Sociological Methodology 13, S. 290 - 312.

Thielsch, M. T. \& Moshagen, M. (2011). Erfassung visueller Ästhetik mit dem VisAWI. In H. Brau, A. Lehmann, K. Petrovic \& M. C. Schroeder (Hrsg.), Usability Professionals 2011, S. 260-265. Stuttgart: German UPA e.V.

Tractinsky, N. (1997). Aesthetics and Apparent Usability: Empirical Assessing Cultural and Methodological Issues. CHI'97 (http://www.acm.org/sigchi/chi97/proceedings/paper/nt.htm).

Tractinsky, N., Katz, A.S., Ikar, D. (2000). What is beautiful is usable. Interacting with Computers 13, S. 127-145.

Tuch, A.; Roth, S.; Hornbaek, K.; Opwis, K. \& Bargas-Avila, J. (2012). Is beautiful really usable? Toward understanding the relation between usability, aesthetics, and affect in HCI. Computers in Human Behavior 28(5), S. 1596-1607.

\section{Kontaktinformationen}

Martin Schrepp: martin.schrepp@sap.com, Kerstin Eva Müller: kerstin.eva.mueller@sap.com 